\title{
Effectiveness of Dental Training in Terms of Anxiety Control Among Dental Students-A Quasi Experimental Study
}

\author{
Yashfika Abdul Bari*, Syeda Maliha Waqar, Saqif Nasir, Khadijah Abid, Maryam Younus, Faran Farooq and \\ Ayesha Rafique
}

Dow University of Health Sciences, Pakistan

Submission: February 01, 2019; Published: February 19, 2019

*Corresponding author: Yashfika Abdul Bari, Dow University of Health Sciences, Karachi, Pakistan

\begin{abstract}
The purpose of the study is to evaluate the effectiveness of dental school training in diminishing dental treatment anxiety among dental students of Dow University of Health Sciences OJHA hospital. The study included first year students who were given questionnaire based on Norman Corah Scale. The students were again given same questionnaire in their final year. The scores were calculated in first and final year of their dental school. Data was analyzed using SPSS version 23. Paired t-test was used to compare pre and post exposure dental anxiety. The results showed that pre anxiety score at first year was $10.08 \pm 3.30$ which significantly reduced as $5.06 \pm 1.57$ at final year after educational exposure $(\mathrm{p}<0.05)$.
\end{abstract}

Keywords: Dental anxiety; Dental students; Tell show do method; Norman corah scale

\section{Introduction}

Dental anxiety is a term use to describe the feeling of fear from seeking dental treatment. It is a challenging issue encountered by dental practitioners in dental clinics. It is one of the reasons for neglecting oral health care since decades [1,2]. It is important to measure among future dental health care providers to account anxiety control measures worldwide. The delay in dental treatment due to dental fear leads to various irreversible disease modalities. The globally increasing fear from seeking dental treatment should be reported in order to provide anxiety control methods. It is seen commonly in majority of the population irrespective of their age [3]. Several studies confirmed existence of dental anxiety globally [4-6] whereas with estimate of 6-15\% globally for patients who avoid dental care because of high levels of dental anxiety and dental phobia [7].

Serra-Negra J et al. [8] in the year 2012 studied dental anxiety among dentists and their patients and it was found that $72.5 \%$ patients suffered dental anxiety and $27.5 \%$ dentists also had dental anxiety. Storjord HP et al. [9] in the year 2014 compared dental fear among students of dentistry, psychology and biology. However, among all three, dental students were least who suffered from dental fear whereas this study also compared anxiety levels between preclinical and post clinical period which was significantly reduced in the last year of dental education.
It is also very important for dental health care providers to report factors causing anxiety in order to control them in future. There are variety of factors that could aggravate their dental fear. Different studies were carried out to find what causes them anxiety. In 1982 and 1997 it was found that injections were the most common anxiety causing factor in dental students whereas the sound of drill was second most anxiety provoking factor $[10,11]$.

Worldwide there is scarcity of recent statistics showing dental anxiety among dental students and variation in their anxiety levels during different years of education. The aim of this study is to determine frequency of dental anxiety among dental students in the first year of dental education followed to their final year where they carried out dental treatment on their own. When dental care provider will be under dental anxiety, it will be very difficult for the dental practitioner to treat their patients with utmost care, hence compromising the patient care.

\section{Materials and Methods}

It is a quasi-experimental study. All the first year students who were enrolled in the course were included in the study in the year 2014 whereas students who were already diagnosed for anxiety disorders or taking any anxiolytics, having any death or emotional trauma last 7 months were excluded in order to control bias. They 
were given questionnaire after 1 month of their enrollment. As the students were included in the study right after their admission to the dental school, it was supposed that there was no dental exposure prior to their enrollment. The same questionnaire was given to same students at the end of their final year of dental school in the year 2018 just before the start of house job assuming they had exposure of dental equipment's and practicing tell, show, do (TSD) method [12,13] in the past years. The students who were already diagnosed for anxiety disorder or taking any anxiolytics, having any death or emotional trauma last 7 months were excluded in order to control bias.

Every year 100 students are enrolled in Dr. Ishrat-ul-Ebad Khan Institute of Health Sciences (DIKIOHS), Karachi, Pakistan for gaining dental education. In DIKIOHS, from the third year of dental school, students start their clinical journey which includes exposing to variety of dental treatments like giving injections, undergoing extractions, taking x-rays, providing scaling of the teeth, carrying out tooth preparations and dealing with all different kind of patients.

\section{Questionnaire}

The questionnaire contained no socio-demographic details and was filled by the students of first year of dental school Table 1: Comparison of pre \& post anxiety score. anonymously. The questionnaire contained 2 parts namely, the questions related to Norman Corah Scale and second part was related to other dental anxiety assessment factors. The anxiety level was calculated based on Norman Corah DAS system (attached as annexure).

\section{Statistical analysis}

Data was analyzed using SPSS version 23. Quantitative variables were presented as mean and standard deviation. Qualitative variables were presented as frequencies and percentages. Paired t-test was used to compare pre and post exposure dental anxiety and dental concern assessment. P-value $\leq 0.05$ was taken as significant.

\section{Results}

There were 100 dental students who filled the questionnaire and during the years 3 students left and 5 failed, leaving 97 dental students for post exposure questionnaire fill up. The mean age of dental students was reported as $22.30 \pm 8.45$ years. Majority of the students were females (57\%) as compared to males (43\%). The pre anxiety score at first year was noted as $10.08 \pm 3.30$ which significantly reduced as $5.06 \pm 1.57$ at final year after educational exposure $(\mathrm{p}<0.05)$ (Table 1).

\begin{tabular}{|c|c|c|c|c|}
\hline \multicolumn{2}{|c|}{ Pre-Anxiety Score at First Year } & \multicolumn{2}{|c|}{ Post Anxiety Score at Final Year After Educational Exposure } & P-value \\
\hline Mean & SD & Mean & SD & 0.001 \\
\hline 10.08 & 3.3 & 5.06 & 1.57 & \\
\hline
\end{tabular}

Majority of the students had moderate anxiety (42\%), followed by mild anxiety (33\%), high anxiety (15\%) and severe anxiety (10\%) at first year. Whereas post exposure, majority of the students had mild anxiety (87\%) followed by moderate anxiety (5\%) and none of the students had high or severe anxiety at final year. (Figure 1).

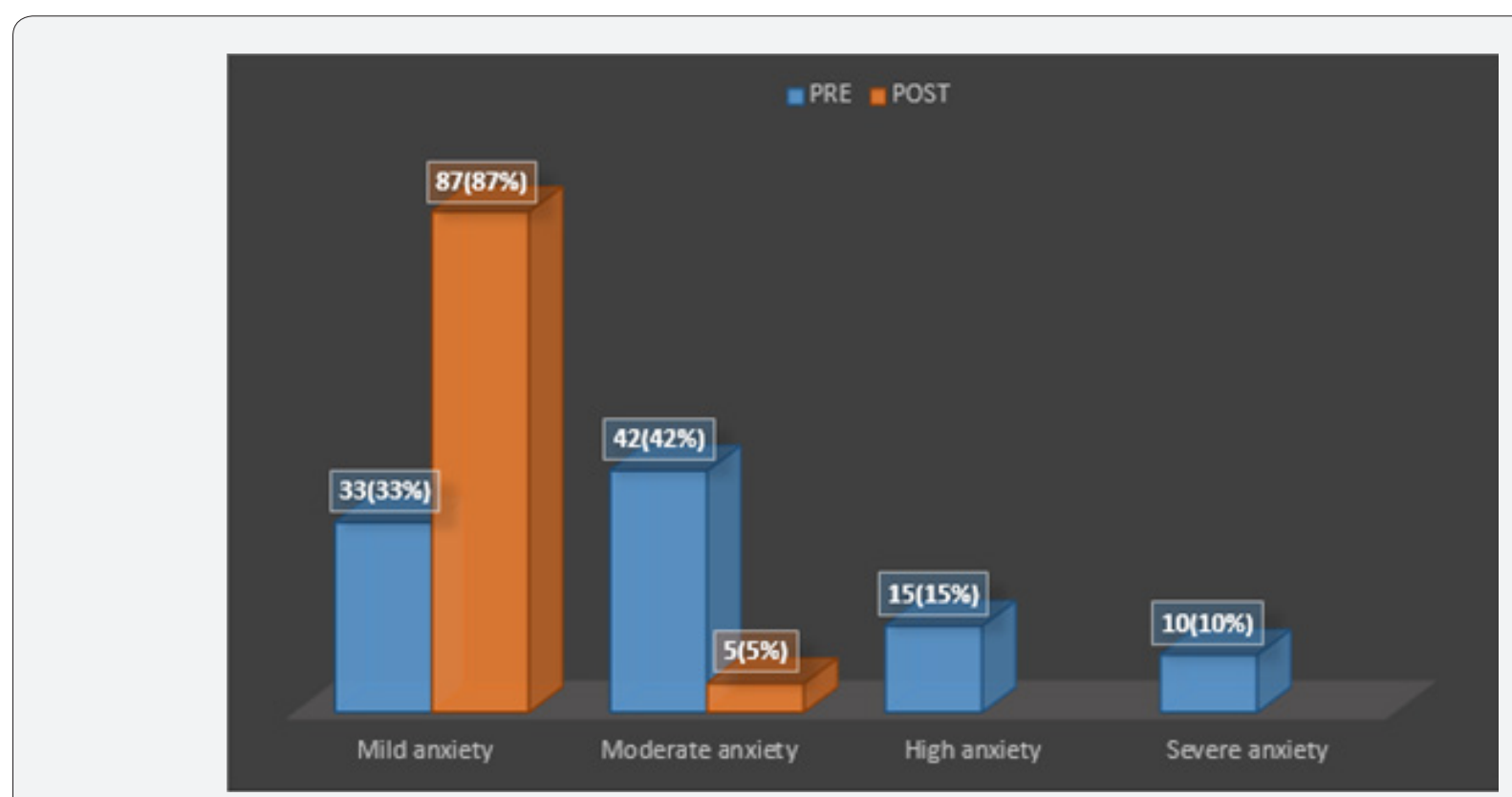

Figure 1: Frequency distribution of pre and post anxiety level.

Dental concern was assessed among these students at first and final year. The factors such as; Sound or vibration of the drill, injection, gagging for example during impressions of the mouth, jaw gets tired, root canal treatment, probing to assess gum 


\section{Advances in Dentistry \& Oral Health}

disease, rubber dam, financial restrain about the dental treatment \& embarrassment about own dental hygiene showed significant difference at final year $(\mathrm{p}<0.05)$, whereas anxiety related to

intra oral radiograph almost remains same at final year $(\mathrm{p}>0.05)$ (Figure 2-4) (Table 2).

Corah's Dental Anxiety Scale, Revised (DAS-R)

Name Date

\section{Norman Corah's Dental Questionnaire}

1. If you had to go to the dentist tomorrow for a check-up, how would you feel about it?

a. I would look forward to it as a reasonably enjoyable experience

b. I wouldn't care one way or the other.

c. I would be a little uneasy about it.

d. I would be afraid that it would be unpleasant and painful.

e. I would be very frightened of what the dentist would do.

2. When you are waiting in the dentist's office for your turn in the chair, how do you feel?

a. Relaxed.

b. A little uneasy

c.

d. Anxious.

e. So anxious that I sometimes break out in a sweat or almost feel physically sick.

3. When you are in the dentist's chair waiting while the dentist gets the drill ready to begin working on your teeth, how do you feel?

a. Relaxed.

b. A little uneasy.

c. Tense

d. Anxious.

e. So anxious that I sometimes break out in a sweat or almost feel physically sick.

4. Imagine you are in the dentist's chair to have your teeth cleaned. While you are waiting and the dentist or hygienist is getting out the instruments which will be used to scrape your teeth around the gums, how do you feel?

a. Relaxed.

b. A little uneasy.

c. Tense,

d. Anxious.

e. So anxious that I sometimes break out in a sweat or almost feel physically sick.

Figure 2:

Scoring the Dental Anxiety Scale, Revised (DAS-R)

(this information is not printed on the form that patients see) $\mathrm{a}=1, \mathrm{~b}=2, \mathrm{c}=3, \mathrm{~d}=4, \mathrm{e}=5 \quad$ Total possible $=20$

Anxiety rating:

- $9-12=$ moderate anxiety but have specific stressors that should be discussed and managed

- $13-14=$ high anxiety

- $15-20$ = severe anxiety (or phobia). May be manageable with the Dental Concerns Assessment but might require the help of a mental health therapist.

Figure 3:

Table 2: Comparison of dental concern assessment before and after exposure.

\begin{tabular}{|c|c|c|c|c|c|}
\hline \multirow{2}{*}{ Dental Concern Assessment } & \multicolumn{2}{|c|}{ Pre-Exposure } & \multicolumn{2}{|c|}{ Post Exposure } & \multirow{2}{*}{ P-value } \\
\hline & Mean & SD & Mean & SD & \\
\hline Sound or vibration of the drill & 2.08 & 0.73 & 1.18 & 0.31 & 0.001 \\
\hline Injection & 2.44 & 0.81 & 1.55 & 0.74 & 0.001 \\
\hline Gagging for example during impressions of the mouth & 2.16 & 0.8 & 2.09 & 0.82 & 0.013 \\
\hline Intra oral radiograph & 1.52 & 0.85 & 1.55 & 0.74 & 0.788 \\
\hline Jaw gets tired & 2.15 & 0.79 & 1.52 & 0.73 & 0.001 \\
\hline Embarrassing oral hygiene & 1.85 & 0.85 & 2.09 & 0.81 & 0.039 \\
\hline Root canal treatment & 2.15 & 0.94 & 1.05 & 0.22 & 0.001 \\
\hline Probing to assess gum disease & 1.79 & 0.91 & 1.05 & 0.22 & 0.001 \\
\hline
\end{tabular}




\begin{tabular}{|c|c|c|c|c|c|}
\hline Rubber Dam & 1.9 & 0.94 & 1.05 & 0.22 & 0.001 \\
\hline I am worried about the cost of the dental treatment I may need & 2.28 & 0.92 & 1.05 & 0.22 & 0.001 \\
\hline
\end{tabular}

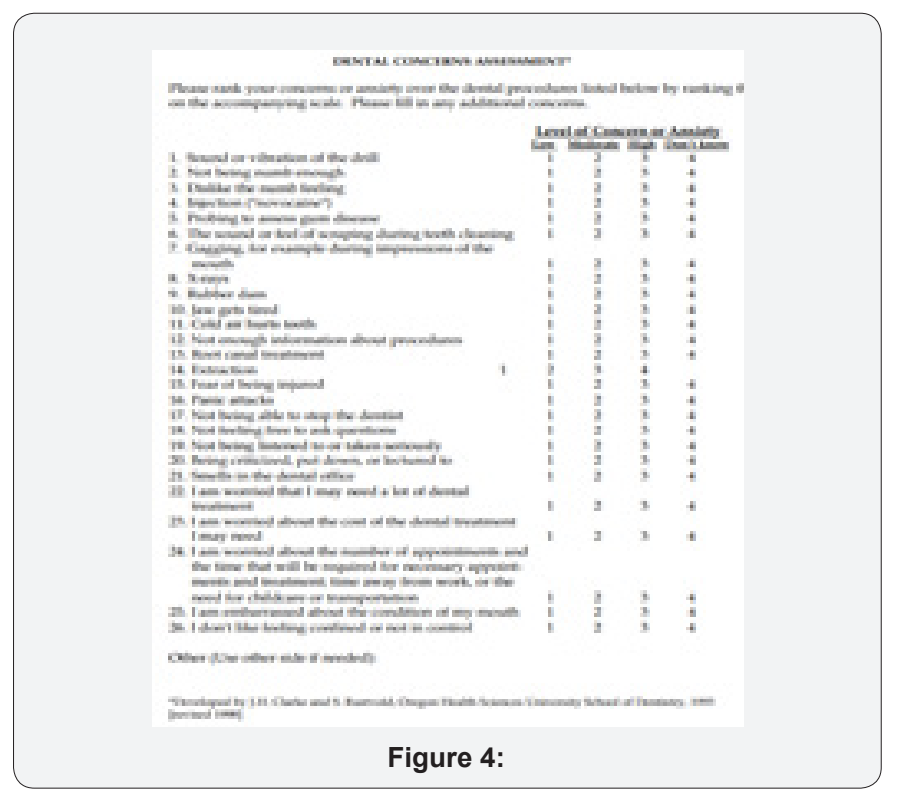

\section{Discussion}

In the present study we have evaluated the dental anxiety among dental students at first and final year and the assessed the dental concerns among them. The outcomes of the present study should that dental students even were afraid regarding dental processes at initial years of the training. A study conducted at Brazil showed that $27.5 \%$ of the dental students suffered from dental phobia [8]. In the present study we have evaluated the DAS at first year as $10.08 \pm 3.30$ which significantly reduced after dental training at final year as $5.06 \pm 1.57(\mathrm{p}<0.05)$. In a similar study conducted by Donka GK et al., the mean DAS was evaluated among 1st year students as $8.37 \pm 0.26$ \& final year students as $7.82 \pm 0.16(\mathrm{p}<0.01)$, which showed that phobia regarding dental procedures significantly decreased with each successive year of dental training $(\mathrm{p}<0.01)$ [14]. Another study conducted in Israel reported that students who were in their 3rd year of dental education had mean dental anxiety level of $9.00 \pm 2.87$ during their 3rd year period which was reduced to $7.67 \pm 2.58$ in the 6 th year of dentistry [15]. A study conducted at Saudi Arab, identified the association of dental fear and anxiety among preclinical and clinical students, which showed significantly higher levels of anxiety scores and dental fear among preclinical students as compared to clinical students $(\mathrm{p}<0.05)$ [16]. Hence enhancing knowledge, education and practice favorably influence the dental anxiety among students.

A longitudinal study was conducted to find out the difference affecting anxiety levels between pre and post clinical experience and it was confirmed that fear of needle was most common among dental students which was significantly reduced in their clinical period after they were continuously exposed to stimulus [15]. There were two more studies carried out by Acharya S et al. [17] in the year 2010 and Al Omari WM et al. [18] in the year 2009 proving that injections caused significant dental anxiety followed by sound of the drill. Recently there were only two studies by Thomas M et al. [19] in 2016 and Ali S et al. [20] in 2015 confirming the correlation between dental needles and stimulating dental anxiety respectively. In the present study, at first year the anxiety score was highest for the fear of injection which significantly decreases at final year. The fear regarding intra oral radiographs remains same at final year. In another similar study showed that fear of injection is one of the most common hindrances in seeking good dental care. Most likely, this can be due to "drill" and "anesthetic needle" are common clinical procedures that can invoke pain [21].

\section{Conclusion}

The importance of dental health prevention protocols \& programs can be implicated in the control \& reduction of dental anxiety. It can be concluded that TSD Method or exposure therapy for reducing dental anxiety among population can be very effective in order to provide great patient care. The change can be reported as anxiety control measures which can further become anxiety control protocol to be followed in every dental hospital setup in order to provide best patient health care and controlling patient's dental anxiety by giving them exposure therapy.

\section{Acknowledgment}

We are immensely grateful to Dr. Mohammed Tariq Kareem, the head of department of Research Evaluation Unit, College of Physicians and Surgeons for sharing their pearls of wisdom and insights as a mentor and supervisor during this research.

\section{References}

1. Eli I (1993) Dental anxiety: a cause for possible misdiagnosis of tooth vitality. International endodontic journal 26(4):251-253.

2. Freeman R (1998) A psychodynamic theory for dental phobia. Br Dent J 184(4):170-172.

3. Yoshida T, Milgrom P, Mori Y, Nakai Y, Kaji M, et al. (2009) Reliability and cross-cultural validity of a Japanese version of the Dental Fear Survey. BMC Oral Health 9(1):17.

4. Berggren U, Meynert G (1984) Dental fear and avoidance: causes, symptoms, and consequences. J Am Dent Assoc 109(2): 247-251.

5. Corah NL, Gale EN, Illig SJ (1978) Assessment of a dental anxiety scale. J Am Dent Assoc 97(5): 816-819.

6. Stabholz A, Peretz B (1999) Dental anxiety among patients prior to different dental treatments. Int Dent J 49(2): 90-94.

7. Eli I, Uziel N, Blumensohn R, Baht R (2004) Modulation of dental anxiety-the role of past experiences, psychopathologic traits and individual attachment patterns. Br Dent J 196(11): 689-694.

8. Serra-Negra J, Paiva SM, Oliveira M, Ferreira E, Freire-Maia F, et al. (2012) Self-reported dental fear among dental students and their patients. Int J Environ Res Public Health 9(1): 44-54.

9. Storjord HP, Teodorsen MM, Bergdahl J, Wynn R, Johnsen JA (2014) Dental anxiety: a comparison of students of dentistry, biology, and psychology. Journal of multidisciplinary healthcare 7:413-418. 
10. Rao A, Sequeire P, Peter S (1997) Characteristics of dental fear amongst dental and medical students. Indian J Dent Res 8(4): 111-114.

11. Wardle J (1982) Fear of dentistry. British Journal of Medical Psychology 55(2):119-126.

12. Barenie JT, Ripa LW (1977) The use of behavior modification techniques to successfully manage the child dental patient. J Am Dent Assoc 94(2): 329-334.

13. Carson P, Freeman R (1998) Tell-show-do: reducing anticipatory anxiety in emergency pediatric dental patients. International Journal of Health Promotion and Education 36(3): 87-90.

14. Kirova DG (2011) Dental anxiety among dental students. Journal of IMAB 17(2): 137-139.

15. Peretz B, Mann J (2000) Dental anxiety among Israeli dental students: a 4-year longitudinal study. Eur J Dent Educ 4(3): 133-137.

16. Hakeem B, Al Shaar A, Qobaly A. Self-assessment of Dental Anxiety and Fear among Dental Students in a Saudi Arabian College.
17. Acharya S, Sangam DK (2010) Dental anxiety and its relationship with self-perceived health locus of control among Indian dental students. Oral Health Prev Dent 8(1): 9-14.

18. Al-Omari WM, Al-Omiri MK (2009) Dental anxiety among university students and its correlation with their field of study. J Appl Oral Sci 17(3): 199-203.

19. Thomas M, Kumar V, Sooraparaju SG, Mathew T, Kumar A, et al. (2016) Dental anxiety among dental, medical, and nursing Students in India and its correlation with their field of study. Journal of International Oral Health 8(8): 860-864.

20. Ali S, Farooq I, Khan SQ, Moheet IA, Al-Jandan BA, et al. (2015) Self-reported anxiety of dental procedures among dental students and its relation to gender and level of education. Journal of Taibah University medical sciences 10(4): 449-453.

21. Gunjal S, Pateel DGS, Parkar S (2017) Dental Anxiety among Medical and Paramedical Undergraduate Students of Malaysia. International Journal of Dentistry 2017: 5.

\section{Your next submission with Juniper Publishers will reach you the below assets}

- Quality Editorial service

- Swift Peer Review

- Reprints availability

- E-prints Service

- Manuscript Podcast for convenient understanding

- Global attainment for your research

- Manuscript accessibility in different formats

( Pdf, E-pub, Full Text, Audio)

- Unceasing customer service

Track the below URL for one-step submission https://juniperpublishers.com/online-submission.php 